\title{
乙基纤维素接枝聚 $N$-异丙基丙烯酰胺共聚物合成及其胶束化研究
}

\author{
康宏亮 ${ }^{a}$ 刘瑞刚*,a 黄勇*,a,b \\ $\left({ }^{a}\right.$ 中国科学院化学研究所 高分子物理与化学国家重点实验室 北京分子科学中心 北京 100190) \\ ( ${ }^{b}$ 中国科学院理化技术研究所 工程塑料国家工程研究中心 北京 100190)
}

\begin{abstract}
摘要 通过单电子转移 “活性” /可控自由基聚合的方法制备了具有双亲性及温度响应性的乙基纤维素接枝聚 $N$-异丙 基丙烯酰胺共聚物(EC-g-PNIPAm). 通过凝胶渗透色谱、核磁氢谱和红外光谱等对合成的接枝共聚物进行了表征. 我 们发现此反应在混合溶剂四氢呋喃/甲醇的混合溶剂中是活性可控的. EC- $g$-PNIPAm 接枝共聚物能够在选择性溶剂水 中发生自组装现象, 形成稳定的以乙基纤维素为核、聚 $N$-异丙基丙烯酰胺为壳的球形胶束. 并且随着温度的升高, 支 链聚 $N$-异丙基丙烯酰胺发生塌缩使得球形胶束发生收缩.
\end{abstract}

关键词 乙基纤维素; 聚 $N$-异丙基丙烯酰胺; 单电子转移 “活性”/可控自由基聚合; 接枝聚合反应; 温敏性

\section{Synthesis of Ethyl Cellulose Grafted Poly(N-isopropylacrylamide) Copolymer and Its Micellization}

\author{
Kang, Hongliang ${ }^{a} \quad$ Liu, Ruigang*,a Huang, Yong*,a,b \\ ( ${ }^{a}$ State Key Laboratory of Polymer Physics and Chemistry, Beijing National Laboratory of Molecular Sciences, \\ Institute of Chemistry, Chinese Academy of Sciences, Beijing 100190) \\ ( ${ }^{b}$ National Engineering Research Center of Engineering Plastics, Technical Institute of Physics and Chemistry, \\ Chinese Academy of Sciences, Beijing 100190)
}

\begin{abstract}
Thermo-responsive graft copolymers, ethyl cellulose grafting poly( $N$-isopropylacrylamide) (EC-g-PNIPAm), were synthesized by single-electron transfer living radical polymerization (SET-LRP) in THF/methanol mixed solvent. Ethyl cellulose macro-initiators (EC-Br) for SET-LRP were synthesized by the esterification between the hydroxyl groups on EC backbones and 2-bromoisobutyryl bromides. The degree of substitution of the EC-Br macro-initiator was tailored by varying the feeding mole ratio of 2-bromoisobutyryl bromide to the hydroxyl group on ethyl cellulose. Three EC-Br macro-initiators with different $\mathrm{Br}$ substitutions were used as the macro-initiators and it was found that the converting degree of the initiator sites was efficient and the graft density of graft copolymer was controllable. The SET-LRP of NIPAm was efficient that the monomer conversion was above $60 \%$ within $25 \mathrm{~h}$. The linear plot of $\ln \left([M]_{0} /[M]_{\mathrm{t}}\right)$ versus the conversion indicated that the reaction was living and controllable. The graft copolymers were characterized by means of gel permeation chromatography (GPC), ${ }^{1} \mathrm{H}$ NMR and FTIR spectroscopy. GPC peak of the graft copolymer became narrower and shifted to the low elution time with the increase of reactive time. The molecular weight of the side-chain linearly increased with the reaction time. EC-g-PNIPAm copolymers were typical amphiphilic graft copolymers, having the hydrophobic EC backbone and the hydrophilic PNIPAm side chains. They could be self-assembled and form stable micelles in a selective solvent, water. The micelles were in spherical shape with typical diameter around $100 \mathrm{~nm}$. Rod-like and flower shaped particles were also observed, which was probably due to the fusion of the spherical shaped micelles. The structure of the spherical shaped micelles was that the EC backbone collapsed to form the core of the micelles and the hydrophilic PNIPAm chains stayed in the outside of the micelles to stabilize the micelles. Due to the thermoresponsive branch chains of PNIPAm, the micelles could shrink with rising temperature due to the collapses of the PNIPAm side chains.

Keywords ethyl cellulose; poly( $N$-isopropylacrylamide); single-electron transfer living radical polymerization (SET-LRP), graft polymerization; thermoresponsive
\end{abstract}

\section{1 引言}

乙基纤维素(EC)是一种常见的纤维素醚，具有优良 的光和热稳定性. 由于乙基纤维素具有较低的燃点、较 小的吸湿性能及良好的绝缘性能, 使其在薄膜和塑料制
品中得到了广泛的应用. 为了扩大乙基纤维素的应用范 围，可以通过接枝改性，从而引入合成高分子的相关性 能. 传统的纤维素接枝方法为自由基接枝聚合，它虽然 具有操作简单、反应条件温和等特点 ${ }^{[1]}$, 但是存在自由

*E-mail: rgliu@iccas.ac.cn; yhuang@iccas.ac.cn; Tel.: 0086-010-82618573; Fax: 0086-010-82618573.

Received October 22, 2012; published December 18, 2012.

Project supported by the National Natural Science Foundation of China (Nos. 20974114, 21174156) and the Knowledge Innovation Program of the Chinese Academy of Sciences (No. KJCX2-YW-H19).

项目受国家自然科学基金(Nos. 20974114, 21174156)和中国科学院知识创新工程(No. KJCX2-YW-H19)资助. 
基聚合容易导致纤维素主链断链、伴随均聚物的产生, 并且支链长度无法控制等缺点.

活性可控自由基聚合物的发现和发展，则克服了传 统自由基聚合的缺点. 其中原子转移自由基聚合反应 (ATRP) 是近年来研究较多, 应用较广的活性可控自由 基聚合反应 ${ }^{[2]}$ ，已广泛应用于合成均聚物、嵌段共聚

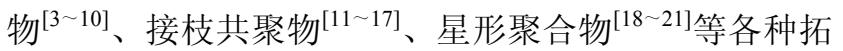
扑结构的高分子材料. Carlmark 等 ${ }^{[22 \sim 24]}$ 首先报道用 ATRP 对纤维素纤维表面进行接枝改性, 以改善纤维素 纤维与其复合材料基体材料的结合能力. 近年来, ATRP 已被用于合成结构明确可控的纤维素接枝共聚物 ${ }^{[25 ~ 41]}$. 这些结构明确的纤维素接枝共聚物在特定条件下可以 进行自组装, 可以用作药物或基因载体, 在药物靶向可 控释放和基因转染等领域有潜在的用途 ${ }^{[38 \sim 44]}$. 江明 等 ${ }^{[45 ~ 47]}$ 合成了具有 $\mathrm{pH}$ 响应特性的羟乙基纤维素接枝聚 丙烯酸(HEC- $g$-PAA) 共聚物, 该接枝共聚物在 $\mathrm{pH}<3$ 时 可以通过自组装形成球形胶束, 进而通过 PAA 核的交 联, 可以固定所制备胶束的结构. 这种胶束溶液通过 $\mathrm{pH}$ 值的改变, 可以实现由聚合物纳米球向中空心球的 可逆转变. 这种胶束还具有离子响应的特性. 进而他们 又合成了无规共聚支链结构的羟乙基纤维素接枝聚 $[N-$ 异丙基丙烯酰胺- $c o$-丙烯酸][HEC- $g$-(PNIPAAm\&PAA)]共 聚物. 该接枝共聚物具有 $\mathrm{pH}$ 和温度的双重响应特性, 可以形成具有双层到三层结构的胶束 ${ }^{[48]}$. 袁金颖课题 组 ${ }^{[31]}$ 结合 ATRP 和开环聚合(ROP)两种方法, 合成了具 有双支链结构的乙基纤维素接枝聚甲基丙烯酸二甲氨 基乙酯聚己内酯(EC- $g$-PDMAEMA- $g$-PCL). 这种共聚 物的独特支链结构使其在水溶液中可以以单分子胶束 状态存在, 当溶液的 $\mathrm{pH}$ 值改变时, 单分子胶束又可以 聚集形成较大的胶束聚集体. 我们课题组在纤维素接枝 共聚物的活性可控制备方面开展了一系列的工作, 成功 合成了多种主侧链结构的纤维素接枝共聚物, 如乙基纤 维素接枝聚苯乙烯(EC- $g-\mathrm{PS})^{[42]}$ 、乙基纤维素接枝聚甲基

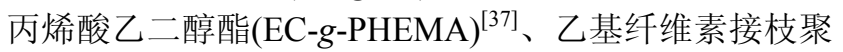
丙烯酸(EC-g-PAA $)^{[49]}$ 、乙基纤维素接枝聚甲基丙烯酸聚 乙二醇单甲醚酯 $[E C-g-P(P E G M A)]^{[39]}$ 、乙基纤维素接枝 聚甲基丙烯酸二乙氨基乙酯(EC- $g$-PDEAEMA) $)^{[41]}$ 等. 根 据主侧链性质的不同, 纤维素接枝共聚物可以在选择性 溶剂中自组装形成不同的结构. 如 EC- $g$-PAA 共聚物随 着支链 PAA 的密度的增加, 其胶束形态可以由球形胶 束转变为短棒状的组装体. 通过调节支链结构, 将具有 温度或 $\mathrm{pH}$ 响应性的聚合物接枝到纤维素主链上, 可以 得到具有响应性的接枝共聚物. 我们以温敏性的差丙基 纤维素(HPC)为主链, 通过 ATRP 合成了具有 $\mathrm{pH}$ 和温度 双重刺激响应性的羟丙基纤维素接枝聚甲基丙烯酸 $N, N$-二甲氨基乙酯 (HPC- $g$-PDMAEMA $)^{[40]}$ 和羟丙基纤 维素接枝聚 4-乙烯吡啶 (HPC-g-P4VP) ${ }^{[38]}$ 共聚物. 通过 它们的水溶液的 $\mathrm{pH}$ 和温度的调节, 可以得到不同核壳 结构的胶束 ${ }^{[38,40]}$.
聚 $N$-异丙基丙烯酰胺(PNIPAm)是一种研究较多的 温度响应性高分子，在水溶液中其最低临界溶液温度 (LCST) 约为 $32{ }^{\circ} \mathrm{C}$, 作为智能材料已在凝胶 ${ }^{[50,51]}$ 、渗透 膜 ${ }^{[52]}$ 和微凝胶 ${ }^{[53]}$ 等方面得到应用。通过自由基聚

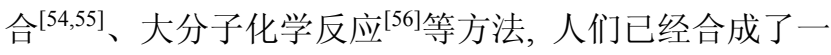
些 PNIPAm 接枝的纤维素接枝共聚物. 但是由于自由基 接枝聚合的产物结构不可控, 并伴随均聚物的产生; 而 大分子反应接枝效率、结构不可控. 因此, 仍需要采用 活性可控的方法, 将温敏性的 PNIPAm 支链可控接枝到 纤维素主链上. 李欢等 ${ }^{[57]}$ 采用可逆加成-断裂链转移聚 合(RAFT)的方法，制备羟丙基纤维接枝 PNIPAm 水凝 胶. 本工作利用单电子转移活性自由基聚合(SET-LRP) 成功合成了具有双亲性和温度响应性的乙基纤维素接 枝聚 $N$-异丙基丙烯酰胺(EC- $g$-PNIPAm)共聚物, 通过接 枝聚合反应动力学数据验证了接枝共聚合反应活性和 可控性，研究了接枝共聚物的自组装行为以及温度响应 特性.

\section{2 结果与讨论}

\subsection{EC-g-PNIPAm 接枝共聚物的合成}

EC- $g$-PNIPAm 接枝共聚物的合成路线如图 1 所示. 首先利用乙基纤维素上剩余的羟基与溴异丁酰溴通过 酯化反应将引发基团引入纤维素主链上，制备大分子引 发剂 EC-Br. 具体的反应条件和大分子引发剂 EC-Br 的 表征见已发表的工作 ${ }^{[30]}$. 大分子引发剂 EC-Br 的活性引 发点的取代度 $\left(D S_{\mathrm{Br}}\right)$ 可以通过改变酯化反应中溴异丁酰 溴与乙基纤维素上羟基的摩尔比来调节. $D S_{\mathrm{Br}}$ 为 $\mathrm{EC}$ 主 链上平均每个葡萄糖单元上的 $\mathrm{Br}$ 官能团个数. 反应条 件与所合成的 $\mathrm{EC}-\mathrm{Br}$ 大分子引发剂的 $D S_{\mathrm{Br}}$ 列于表 1 .
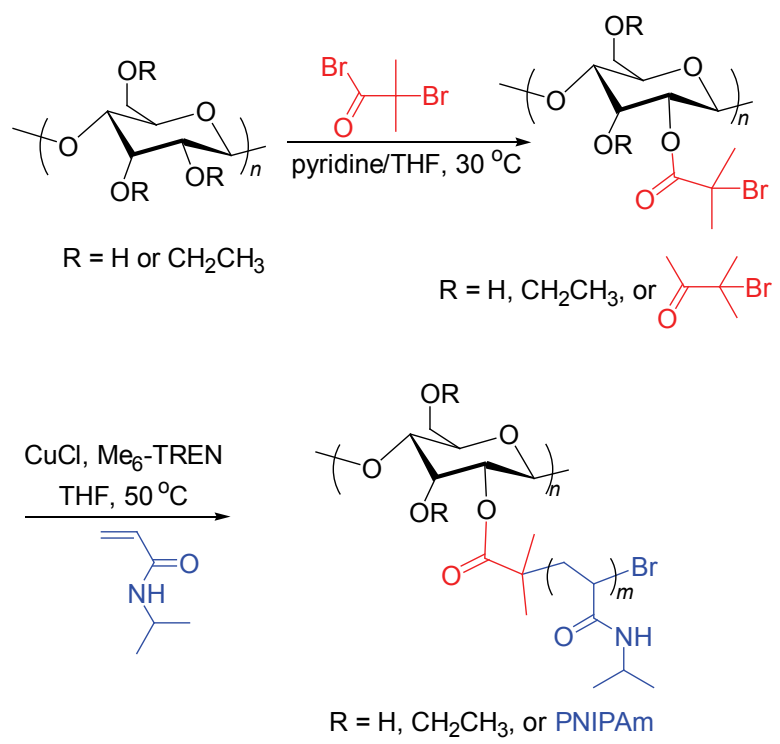

图 1 EC- $g$-PNIPAm 接枝共聚物的合成路线

Figure 1 Synthesis route of EC-g-PNIPAm

三(2-二甲氨基乙基)胺( $\mathrm{Me}_{6}$-TREN)是具有较强配位 
能力的配体, 能够同催化剂协同作用更好的促进 $N$-异 丙基丙烯酰胺的聚合反应 ${ }^{[58]}$. 本文中, 大分子引发剂 $\mathrm{EC}-\mathrm{Br}$ 作为引发剂、 $\mathrm{Cu}(\mathrm{I}) / \mathrm{Me}_{6}-\mathrm{TREN}$ 作为催化剂, 在四 氢呋喃/甲醇 $(35 / 2, V / V)$ 的混合溶剂中 $50{ }^{\circ} \mathrm{C}$ 引发 $N$-异丙 基丙烯酰胺单体的聚合反应，具体的反应条件见表 2 . 文献 ${ }^{[58]}$ 结果显示, 以 $\mathrm{Cu}(\mathrm{I}) / \mathrm{Me}_{6}-\mathrm{TREN}$ 为催化剂, 在醇 溶液(如 2-丙醇和异丁基醇)中进行反应, 可以得到分子 量分布较窄的 PNIPAm. 在反应介质中引入甲醇可以增 加催化剂的溶解度, 有利于接枝聚合反应的进行. EC- $g$-PNIPAm 接枝共聚物的接枝密度通过大分子引发 剂 $\mathrm{EC}-\mathrm{Br}$ 的 $D S_{\mathrm{Br}}$ 控制. 我们采用了 3 个不同取代度的 EC-Br 用于制备不同取代度的 EC- $g$-PNIPAm, 反应条件 和结果见表 2 .

表 1 乙基纤维素大分子引发剂的制备

Table 1 Preparation of EC-Br macro-initiators

\begin{tabular}{ccc}
\hline Mole ratio $^{a}$ & Reaction time/d & $D S_{\mathrm{Br}}{ }^{b}$ \\
\hline $1: 1$ & 2 & 0.04 \\
$2: 1$ & 2 & 0.09 \\
$3: 1$ & 2 & 0.28 \\
$4.5: 1$ & 2 & 0.30 \\
$5: 1$ & 2 & 0.37 \\
\hline
\end{tabular}

${ }^{a}$ The mole ratio of 2-bromoisobutyryl bromides/hydroxyl group on the ethyl cellulose; ${ }^{b}$ Degree of substitution of bromoisobutyryl groups, bromoisobutyryl groups per glucose ring of cellulose.

图 2 为单体的转化率与反应时间及 $\ln \left([\mathrm{M}]_{0} /[\mathrm{M}]_{t}\right)$ 与 单体转化率之间的关系, 其中 $[\mathrm{M}]_{0}$ 为体系中单体起始浓 度, $[\mathrm{M}]_{t}$ 为反应时间 $t$ 时体系中的单体浓度. 图 2 中结果 表明, 在反应进行 $30 \mathrm{~h}$ 内, 单体转化率随反应时间的增 加而线性增加, $\ln \left([\mathrm{M}]_{0} /[\mathrm{M}]_{t}\right)$ 也随单体转化率的增加线 性而增加. 这一现象符合一级动力学反应特征, 说明反 应体系中的增长自由基的浓度在反应过程中是保持不 变的, 接枝聚合反应是活性可控的. 本工作中的单体转 化率与反应的时间关系与文献报道的 $N$-异丙基丙烯酰 胺在甲醇中的 ATRP 反应不同. 在该文献中, 单体转化 率与 $\ln \left([\mathrm{M}]_{0} /[\mathrm{M}]_{t}\right)$ 随反应时间的增加而呈曲线增大 ${ }^{[58]}$, 而本工作中的反应动力学曲线则是线性的. 此外, 在本 工作中, 接枝聚合物反应具有较高的单体转化率, 反应 时间在 $25 \mathrm{~h}$ 内单体转化率均在 $60 \%$ 以上. 所制备的 EC- $g$-PNIPAm 共聚物的 PNIPAm 支链分子量 $\left(M_{\mathrm{n}}\right)$ 可以
依据所得到的共聚物的 ${ }^{1} \mathrm{H}$ NMR 谱图，根据下列公式计 算得到.

$$
M_{\mathrm{n}}=\left[3 \cdot D S_{\text {Ethyl }} A_{\mathrm{d}}\right] /\left(D S_{\mathrm{Br}}\left(A_{\mathrm{a}}-6 A_{\mathrm{d}}\right)\right)
$$

式中, $A_{\mathrm{a}}$ 为接枝共聚物中 PNIPAm 和 $\mathrm{EC}$ 中的 $\mathrm{CH}_{3}$ 中的 氢原子的特征峰 $\left(\delta\right.$ 1.1) 的积分面积, $A_{\mathrm{d}}$ 为支链 PNIPAm 中 $\mathrm{NH}$ 基团中的氢原子特征峰( $\delta 6.0$ )的积分面积(图 5b), $D S_{\mathrm{Br}}$ 是乙基纤维素大分子引发剂中溴异丁酸酯基团的 取代度, $D S_{\text {Ethyl }}$ 是乙基纤维素中乙基的取代度, $M_{\mathrm{NIPAm}}$ 是 单体 $N$-异丙基丙烯酰胺的分子量. 通过计算得到 PNIPAm 支链的分子量与反应时间的关系如图 3. 结果 发现，PNIPAm 支链的分子量是随着反应时间的增加而 线性增加.
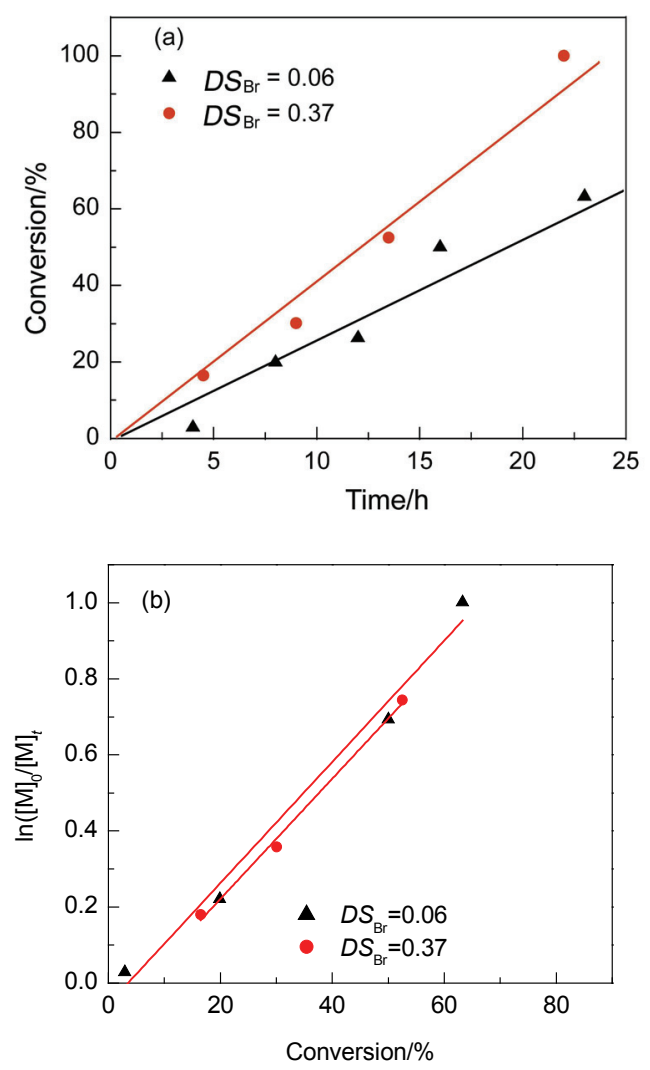

图 2 单体转化率与反应时间 $(\mathrm{a})$ 及 $\ln \left([\mathrm{M}]_{0} /[\mathrm{M}]_{t}\right)$ 与单体转化率(b)之间 的关系曲线

Figure 2 Monomer conversion vs. reaction time (a) and $\ln \left([\mathrm{M}]_{0} /[\mathrm{M}]_{t}\right)$ vs. monomer conversion in $\mathrm{THF} /$ methanol

Reaction parameters, $[\mathrm{M}]:[\mathrm{Br}]:[\mathrm{CuCl}]:\left[\mathrm{Me}_{6}-\mathrm{TREN}\right]=60: 1: 1: 2$; reaction temperature of $50{ }^{\circ} \mathrm{C}$

表 2 四氢呋喃/甲醇 $(35 / 2, V / V)$ 中 SET-LRP 接枝聚合反应条件和所制备的 EC- $g$-PNIPAm 接枝共聚物

Table 2 Details of SET-LRP reaction in THF/methanol $(35 / 2, V / V)$ and the resultant EC-g-PNIPAm copolymers

\begin{tabular}{cccccc}
\hline$D S_{\mathrm{Br}}$ & {$[\mathrm{M}]:[\mathrm{I}]:\left[\mathrm{Cu}^{\mathrm{I}}\right]:\left[\mathrm{Me}_{6}-\mathrm{TREN}\right]$} & Time $/ \mathrm{h}$ & Conversion $/ \%$ & $M_{\mathrm{n}}{ }^{a} /\left(\mathrm{g} \cdot \mathrm{mol}^{-1}\right)$ & $M_{\mathrm{w}} / M_{\mathrm{n}}{ }^{a}$ \\
\hline 0.06 & $30: 1: 1: 2$ & 17 & 48 & $3.22 \times 10^{5}$ & 1.7 \\
0.28 & $30: 1: 1: 1$ & 12 & 15 & $1.60 \times 10^{5}$ & 2.6 \\
0.37 & $60: 1: 1: 2$ & 15 & 8.4 & $1.7 \times 10^{5}$ & 2.4 \\
\hline
\end{tabular}

${ }^{a}$ GPC results of EC-g-PNIPAm copolymers. 


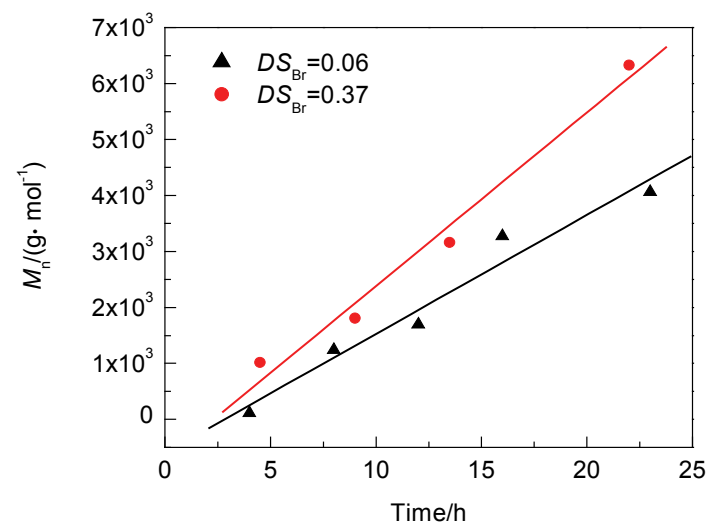

图 3 由核磁氢谱计算的支链的数均分子量

Figure $3 M_{\mathrm{n}}$ of graft chains calculated from ${ }^{1} \mathrm{H}$ NMR spectra

在本文工作中, 合成 EC-g-PNIPAm 共聚物的反应 体系中使用了 $\mathrm{CuCl} / \mathrm{Me}_{6} \mathrm{TREN}$ 作为催化剂, 极性的甲醇 作为溶剂. 由于 $\mathrm{CuCl}$ 在本实验条件下容易发生歧化反 应, 聚合的机理有可能是单电子转移 “活性” 可控自由 基聚合(SET-LRP $)^{[59]}$, 因此依据文献 ${ }^{[60,61]}$ 报道, 设计如 下实验进行验证.

将 $3 \mathrm{~mL}$ 四氢呋喃、 $2 \mathrm{~mL}$ 甲醇与 $\mathrm{CuCl}(0.0115 \mathrm{~g})$ 和 $\mathrm{Me}_{6} \mathrm{TREN}(30 \mu \mathrm{L})$ 混合置于试管中. 作为对比, 另一组 实验用 $\mathrm{CuCl}_{2}(0.0142 \mathrm{~g})$ 替代 $\mathrm{CuCl}$. 将两个样品均通入 $\mathrm{N}_{2}$ 气鼓泡 $30 \mathrm{~min}$, 并且在室温下搅拌. 结果发现, 在 $\mathrm{CuCl} / \mathrm{Me}_{6} \mathrm{TREN}$ 体系中出现了黑色单质 $\mathrm{Cu}$ 沉淀(图 4 中 照片左图), 而 $\mathrm{CuCl}_{2} / \mathrm{Me}_{6} \mathrm{TREN}$ 体系没有沉淀出现(图 4 中照片右图). 由图 4 所示的两种溶液的紫外吸收光谱 结果可知, $\mathrm{CuCl} / \mathrm{Me}_{6} \mathrm{TREN}$ 体系的吸收峰的波长与 $\mathrm{CuCl}_{2} / \mathrm{Me}_{6} \mathrm{TREN}$ 体系吸收峰一致, 说明一价 $\mathrm{Cu}^{(\mathrm{I})}$ 在四氢 呋喃/甲醇混合溶剂中很难稳定存在. 根据图 4 的实验结 果, 可以初步认为本文中的乙基纤维素接枝聚 $N$-异丙 基丙烯酰胺的反应机理应该为 SET-LRP ${ }^{[59 \sim 61]}$.

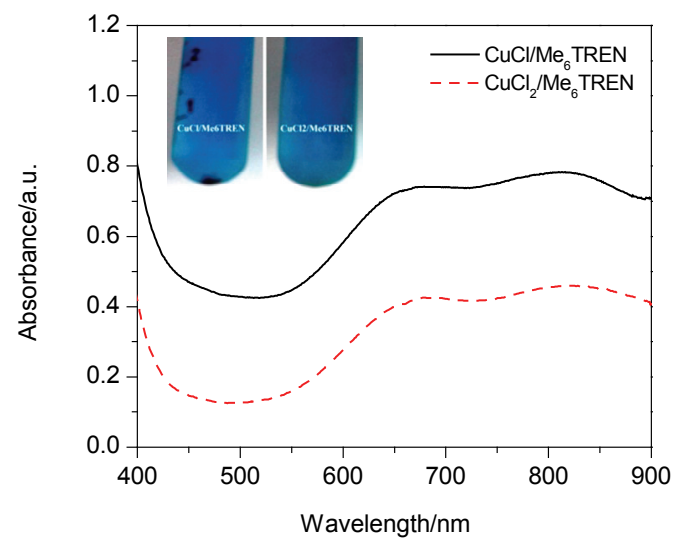

图 4 含有 $\mathrm{CuCl} / \mathrm{Me}_{6} \mathrm{TREN}$ 和 $\mathrm{CuCl}_{2} / \mathrm{Me}_{6} \mathrm{TREN}$ 的四氢呋喃/甲醇混合 溶液的紫外吸收光谱

Figure 4 UV-Vis spectra of $\mathrm{THF} / \mathrm{CH}_{3} \mathrm{OH}$ solutions containing $\mathrm{CuCl} / \mathrm{Me}_{6}$ TREN and $\mathrm{CuCl}_{2} / \mathrm{Me}_{6}$ TREN
图 5 是 $\mathrm{EC}-g$-PNIPAm 接枝共聚物的 GPC 曲线. 接 枝共聚物 GPC 的流出峰随着反应时间的增加变窄并向 低洗脱时间方向移动. 当反应时间在 $15 \mathrm{~h}$ 时, EC- $g$ PNIPAm 接枝共聚物的数均分子量是 $160000 \mathrm{~g} / \mathrm{mol}$, 分 子量分布是 2.6. 反应时间再增加到 $34 \mathrm{~h}$ 时, 数均分子 量为 $319000 \mathrm{~g} / \mathrm{mol}$, 分子量分布变窄为 1.7 . 由此可知这 个体系的聚合反应成功进行, 并且接枝共聚物的分子量 分布较主链乙基纤维素的窄.

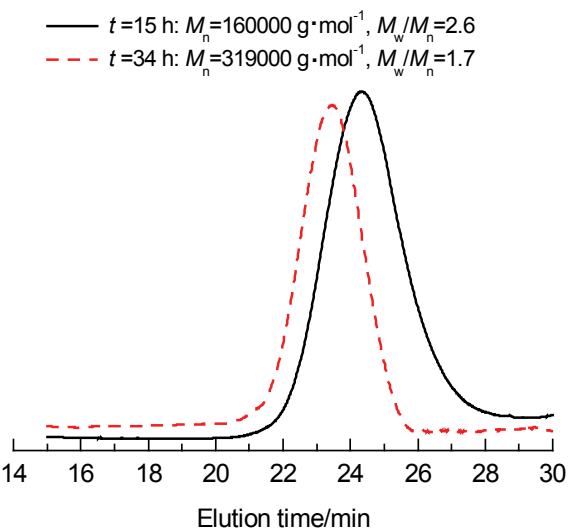

图 5 EC- $g$-PNIPAm 接枝共聚物的 GPC 曲线图

Figure 5 GPC traces of EC-g-PNIPAm copolymer

图 6 为大分子引发剂 $\mathrm{EC}-\mathrm{Br}$ 和接枝共聚物 EC- $g$-PNIPAm 进行核磁氢谱的表征，图 6a 中 EC-Br 的 核磁氢谱中化学位移 $\delta 1.95$ 的特征峰归属于大分子引发 剂中溴异丁酸酯基团中 $\left[\left(\mathrm{CH}_{3}\right)_{2} \mathrm{Br}\right]$ 甲基的峰, $\delta 1.28$ 归属 于乙基纤维素乙基上的甲基的质子峰，宽峰 $\delta 3.0 \sim 5.0$ 归属于乙基纤维素上葡萄糖环上的质子和乙基纤维素 乙基中的亚甲基上的质子峰. 引发点澳异丁酸酯基团的 取代度通过比较 $\delta 1.95$ 和 1.28 处峰的积分计算得到. 接 枝共聚物的支链 PNIPAm 中异丙基上甲基的氢同乙基 纤维素上甲基的氢的峰重合, 位置在 $\delta$ 1.1. 化学位移 $\delta$ 1.5 2.8 归属于 PNIPAm 中 $\left(\mathrm{CH}_{2} \mathrm{CH}\right)_{n}$ 的氢的特征峰. 化 学位移 $\delta 6.0$ 属于 $\mathrm{NH}$ 中氢的特征峰, 其它氢的归属明确 表示在图 $6 \mathrm{~b}$ 中. 由核磁氢谱可以看出化学位移在 $\delta 1.95$ 处的峰消失了, 表明大分子引发剂所有活性的溴引发基 团均有效地引发了 $N$-异丙基丙烯酰胺单体的聚合反应. 图 7 是 EC-Br 大分子引发剂和 EC- $g$-PNIPAm 接枝共聚 物的红外光谱图. 与 $\mathrm{EC}-\mathrm{Br}$ 大分子引发剂的红外光谱比 较, EC- $g$-PNIPAm 接枝共聚物的红外光谱在 $3318 \mathrm{~cm}^{-1}$ 是 $\mathrm{N}-\mathrm{H}$ 的伸缩振动峰, 1536 和 $1673 \mathrm{~cm}^{-1}$ 为酰胺 (I) 和 酰胺(II)的伸缩振动峰, 这些都是 PNIPAm 的特征谱带. 核磁氢谱和红外光谱实验结果均证明我们成功合成了 EC- $g$-PNIPAm 接枝共聚物.

\subsection{EC-g-PNIPAm 接枝共聚物胶束}

EC- $g$-PNIPAm 接枝共聚物是典型的双亲性接枝共 聚物，由亲油性的主链乙基纤维素和在 LCST 温度以下 
(a)

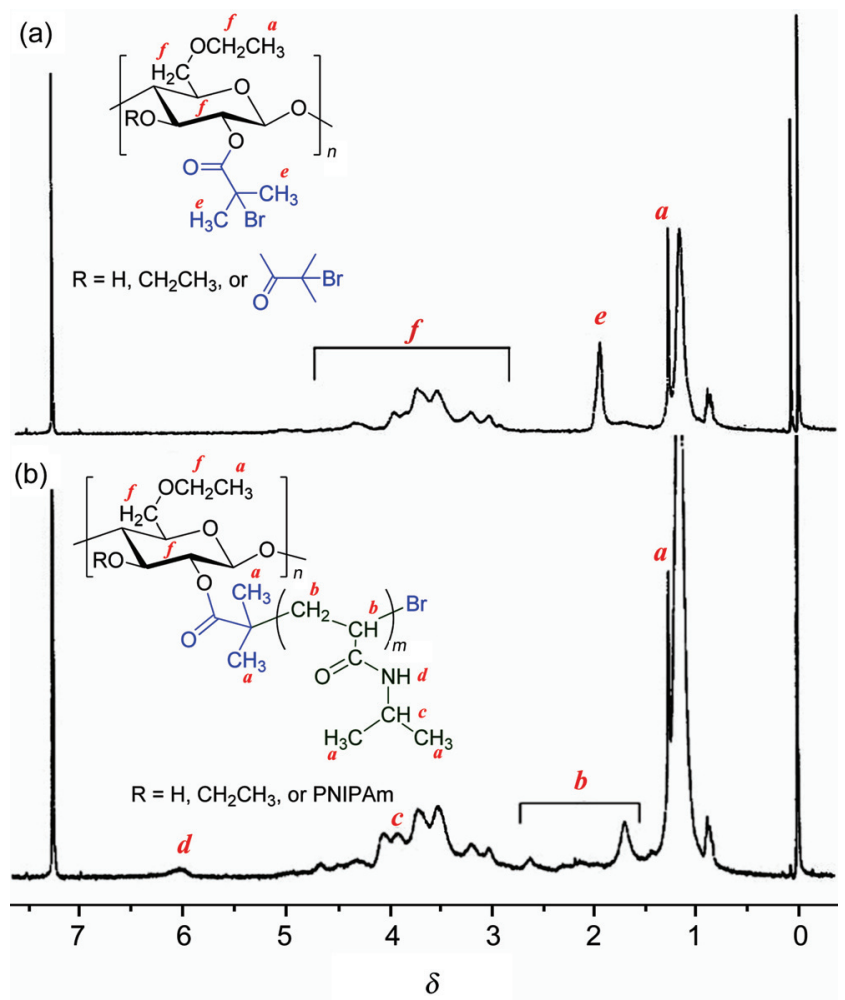

图 $6 \mathrm{EC}-\mathrm{Br}$ (a)和 EC- $g$-PNIPAm 接枝共聚物(b)的核磁氢谱图 Figure $6{ }^{1} \mathrm{H}$ NMR spectra of (a) EC-Br and (b) EC-g-PNIPAm copolymer in $\mathrm{CDCl}_{3}$

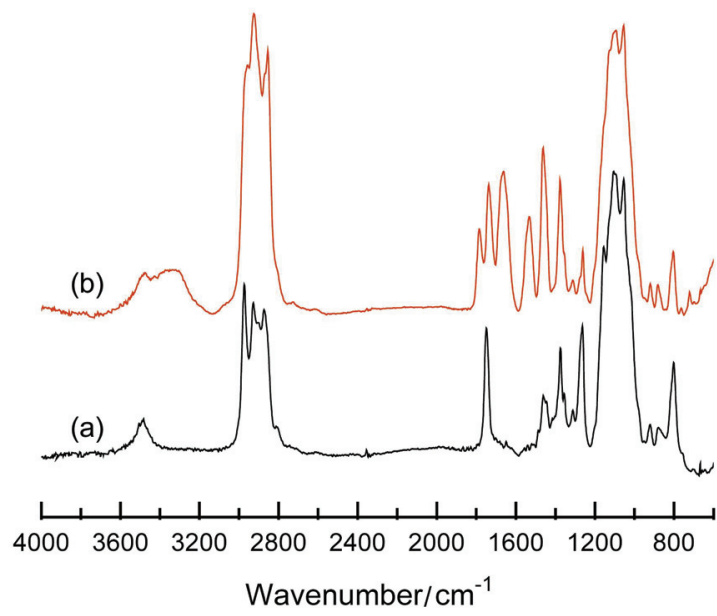

图 7 EC-Br (a)和 EC- $g$-PNIPAm 接枝共聚物(b)的红外光谱图

Figure 7 FTIR spectra of (a) EC-Br and (b) EC-g-PNIPAm copolymer

具有亲水性的聚 $N$-异丙基丙烯酰胺组成. 在选择性溶 剂中这种具有双亲性的接枝共聚物能够发生自组装现 象，可以制备比较稳定的胶束. 水是乙基纤维素主链的 不良溶剂; 而对于支链聚 $N$-异丙基丙烯酰胺而言, 在它 的 LCST 温度以下, 可以很好的溶解在水中 ${ }^{[62]}$. 因此, 将水作为选择性溶剂制备接枝共聚物的胶束. 依据文 献 ${ }^{[63,64]}$ 测定 $\mathrm{EC}_{0.37}-g$-PNIPAm 28 接枝共聚物的临界胶束 浓度为 $1.59 \times 10^{-4} \mathrm{mg} / \mathrm{mL}$.
通过将 $\mathrm{EC}_{0.37}-g$-PNIPAm 28 接枝共聚物溶解在 DMF 溶剂中，然后在水溶液中进行透析得到稳定的胶束，其 中此接枝共聚物的支链密度 0.37 , 每个支链的重复单元 数为 28. 图 8 是制备的接枝共聚物胶束的形貌图. 由图 可知, 多数为球形的胶束, 尺寸大约在 $100 \mathrm{~nm}$; 同时还 可观察到棒状和花朵形状的胶束, 推测原因是在制备 TEM 样品时水分的蒸发过程中, 球形胶束发生了融合 导致的. 由主侧链亲水性的不同可知, 球形胶束是以主 链乙基纤维素塌缩形成核, 亲水性的聚 $N$-异丙基丙烯 酰胺为壳的结构 ${ }^{[65]}$. 聚 $N$-异丙基丙烯酰胺是具有温敏 特性的聚合物, 得到的这种球形的接枝共聚物胶束对温 度是敏感的. 通过 DLS 来表征胶束的尺寸随着温度的 变化行为, 如图 9 所示. 由图 9 可知, 在 $17{ }^{\circ} \mathrm{C}$ 时胶束的 流体力学半径 $\left(<R_{\mathrm{h}}>\right)$ 约为 $130 \mathrm{~nm}$, 随着温度的升高, $<R_{\mathrm{h}}>$ 逐渐减小. 当溶液的温度升高到 $35{ }^{\circ} \mathrm{C}$ 时, $<R_{\mathrm{h}}>$ 下降到约为 $100 \mathrm{~nm}$, 并且 $<R_{\mathrm{h}}>$ 的分布变窄. EC-g-PNIPAm 接枝共聚物这种尺寸随温度的变化不及 超高分子量的聚 $N$-异丙基丙烯酰胺 ${ }^{[66]}$ 和聚 $N$-异丙基丙 烯酰胺凝胶 ${ }^{[67]}$ 随温度变化的明显，主要源于其接枝共 聚物支链的分子量较低, 进而发生较小的体积变化.

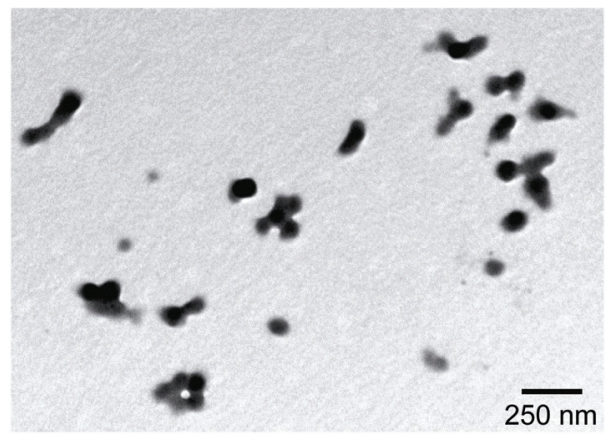

图 $8 \mathrm{EC}_{0.37}-g-\mathrm{PNIPAm} 28$ 的 DMF 溶液经水透析制备的胶束的 TEM 图 Figure 8 Typical TEM image of the micelles prepared from $\mathrm{EC}_{0.37}{ }^{-}$-PNIPAm 28 dialyzed against distilled water from DMF solution

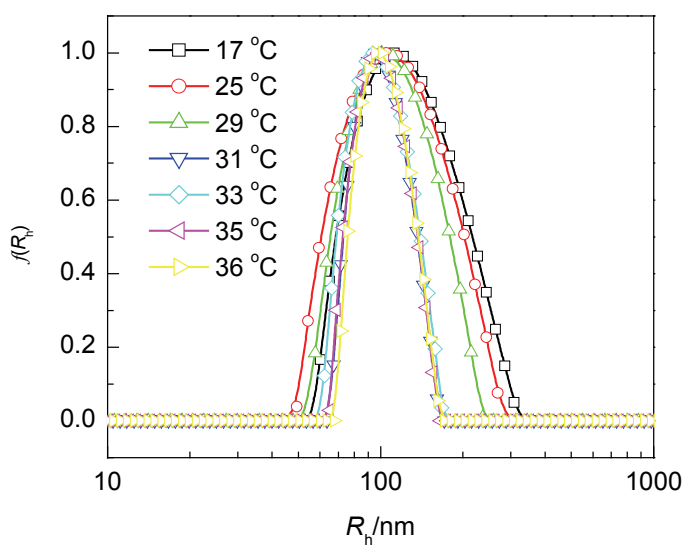

图 $9 \mathrm{EC}_{0.37}-\mathrm{g}$-PNIPAm 28 接枝共聚物胶束在不同温度的水溶液中的流 体力学半径及分布

Figure 9 Typical hydrodynamic radius and its distribution of micelles prepared from $\mathrm{EC}_{0.37-}$-PNIPAm 28 copolymers at different temperature in aqueous solution

Data were collected at the scattering angle of $90^{\circ}$ 


\section{3 结论}

利用乙基纤维素上剩余的羟基与溴异丁酰溴的酯 化反应，制备了用于 SET-LRP 反应的大分子引发剂 $\mathrm{EC}-\mathrm{Br}$, 大分子引发剂的引发点数量的改变通过调整羟 基与溴异丁酰溴的摩尔比. 大分子引发剂 $\mathrm{EC}-\mathrm{Br}$ 在 $\mathrm{CuCl} / \mathrm{Me}_{6}-\mathrm{TREN}$ 催化下, 可以在四氢呋喃/甲醇 $(35 / 2$, $V / V)$ 中引发 $N$-异丙基丙烯酰胺的接枝聚合反应，合成乙 基纤维素接枝聚 $N$ - 异丙基丙烯酰胺共聚物 (EC-g-PNIPAm). 动力学实验结果表明接枝聚合反应是 活性可控的, 可以用于合成结构明确可控的 EC- $g$ PNIPAm 共聚物. 所合成的 EC- $g$-PNIPAm 共聚物具有 双亲特性和温度敏感的性能, 在水溶液中可以自组装形 成主链 EC 为核、支链 PNIPAm 为壳的稳定胶束. 支链 PNIPAm 的温度敏感性赋予了胶束温度响应性, 随着胶 束溶液的温度升高, 胶束的尺寸由于 PNIPAm 壳的塌缩 而减小.

\section{4 实验部分}

\section{1 原料}

$N$-异丙基丙烯酰胺(NIPAm, 98\%, Aldrich), 在正己 烷中重结晶二次后待用, 低温储藏. 乙基纤维素(EC, 沪州化工厂), 乙基取代度为 $2.1, M_{\mathrm{n}}=1.4 \times 10^{5} \mathrm{~g} / \mathrm{mol}$, $M_{\mathrm{w}} / M_{\mathrm{n}}=2.97$, 真空 $50{ }^{\circ} \mathrm{C}$ 干燥 $2 \mathrm{~d}$ 待用. 氯化亚铜 $(\mathrm{CuCl}$, 分析纯, 上海振兴化学试剂公司产品)除去二价铜离子 $\mathrm{Cu}^{2+}$ 后待用. 具体方法如下: 用冰醋酸反复进行浸泡至 上层清液为无色, 过滤除去冰醋酸后用丙酮反复洗涤后 过滤, 所得到的 $\mathrm{CuCl}$ 在室温下真空干燥, 避光保存. 吡 啶和甲醇经无水硫酸镁干燥除水过滤后使用. 三(2-二 甲氨基乙基)胺( $\mathrm{Me}_{6}$-TREN)依据文献[68]的方法由三(2氨基乙基)胺(TREN，98\%，Aldrich)制得. 溴异丁酰溴 (98\%, Aldrich)直接使用. 四氢呋喃(THF)经减压蒸馏后 使用. 氯化铜 $\left(\mathrm{CuCl}_{2}\right.$, 分析纯, 上海振兴化学试剂公司 产品)、萠(pyrene, 99\%, Alfa Aesar)直接使用.

\section{2 接枝聚合反应}

EC-g-PNIPAm 接枝共聚物的合成路线如图 1 所示. 乙基纤维素大分子引发剂(EC-Br)根据以前报道 ${ }^{[30]}$ 的方 法制备. EC-Br 中引发点溴的取代度 $\left(D S_{\mathrm{Br}}\right)$ 通过调节溴异 丁酰溴同乙基纤维素上羟基的摩尔比来实现.

在 $50 \mathrm{~mL}$ 的圆底烧瓶中将 $0.1431 \mathrm{~g}$ 的大分子引发剂 $\mathrm{EC}-\mathrm{Br}(0.2 \mathrm{mmol}$ 的引发点) 充分溶解在 $30 \mathrm{~mL}$ 的 THF 中, 加入 $1.360 \mathrm{~g}(12 \mathrm{mmol}) \mathrm{NIPAm}$ 单体后, 通 $\mathrm{N}_{2}$ 气除氧 20 30 min. 然后加入经过除氧的 $\mathrm{CuCl}(0.02 \mathrm{~g}, 0.2$ $\mathrm{mmol}$ )和 $\mathrm{Me}_{6}$-TREN (0.092 $\left.\mathrm{g}, 0.4 \mathrm{mmol}\right)$ 的 THF/甲醇 $(5 / 2$, $V / V)$ 溶液 $5 \mathrm{~mL}$, 溶液立即变为绿色. 继续通 $\mathrm{N}_{2}$ 气 $10 \mathrm{~min}$, 密封, 然后将圆底烧瓶置于 $50{ }^{\circ} \mathrm{C}$ 的油浴锅中进行反应. 间隔一定的时间用针头取出一定数量的样品, 用于反应
动力学实验. 反应一段时间后，将反应混合物暴露于空 气中来停止反应. 将反应后的混合物通过装有氧化铝的 层析柱, 以去除体系中的催化剂. 得到的产物溶液浓缩 后转移至截留分子量为 $3.5 \mathrm{kDa}$ 的透析袋中, 用蒸馏水 透析 $4 \mathrm{~d}$ 后冷冻干燥得到 EC- $g$-PNIPAm 共聚物.

\subsection{EC- $g$-PNIPAm 接枝共聚物的胶束的制备}

将 EC-g-PNIPAm (0.005 g) 接枝共聚物在 DMF $(10 \mathrm{~mL})$ 充分溶解后, 将所得到的溶液在蒸馏水中透 析(截留分子量为 $3.5 \mathrm{kDa}$ ), 得到 EC- $g$-PNIPAm 胶束.

\section{4 分析与测试}

单体的转化率通过称重法测定. 准确称取一定量的 聚合溶液, 加入 THF 稀释, 通过含有氧化铝的层析柱, 得到的溶液旋转蒸发掉一部分溶剂, 然后倒入截留分子 量为 $3.5 \mathrm{kDa}$ 的透析袋, 用蒸馏水透析 $3 \mathrm{~d}$, 然后再冷冻 干燥, 称重, 计算单体转化率.

聚合物的相对分子质量及其分布用 Waters 凝胶渗 透色谱仪测定, 输液百: Waters HPLC 515 pump; 检测 器: Waters 2410 Refractive Index Detector; 色谱柱: WAT044231, WAT044234, WAT044222, WAT044210; 测试温度: $30{ }^{\circ} \mathrm{C}$; 流动相: $\mathrm{THF}$; 流速: $1.0 \mathrm{~mL} / \mathrm{min}$; 用 聚苯乙烯标样对色谱柱进行校正.

${ }^{1} \mathrm{H}$ NMR 测试采用 Bruker $400 \mathrm{MHz}$ 核磁共振仪, 溶 剂: $\mathrm{CDCl}_{3}$ ，四甲基硅为内标.

傅立叶转换红外光谱(FT-IR)在 Brucker-Equinox 55 红外光谱仪上测试. 接枝共聚物溶于氯仿中, 在 $\mathrm{NaCl}$ 盐片上涂膜，红外灯下烘烤去除溶剂后测试.

透射电子显微镜(TEM) 观察在 Hitachi H-800 上进行, 加速电压 $100 \mathrm{kV}$. 样品制备: 将 $5 \mu \mathrm{L}$ 的 EC-g- PNIPAm 接枝共聚物胶束溶液滴加到涂有碳膜的铜网上，室温干 燥后用于 TEM 观察.

动态激光光散射(DLS)实验在 ALV/SP-150 激光光 散射仪上进行, 配有 ALV-5000 multi- $\tau$ 数字时间相关仪. ADLAS DPY 425 II 固态激光器为光源, 输出功率为 400 $\mathrm{mV}(\lambda=632.8 \mathrm{~nm})$. DLS 实验散射角为 $90^{\circ}$, 温度为 25 ${ }^{\circ} \mathrm{C}$. 采用 CONTIN 方法分析强度相关函数得到样品的表 观流体力学半径 $\left(<R_{\mathrm{h}}>\right)$ 及其分布.

紫外吸收光谱使用 Shimadzu 1601PC 紫外分光光度 计测定.

临界胶束浓度通过稳态苂光光谱测定. 稳态稳态苂 光光谱在 Cary Eclipse 苂光光度计上测定. 使用萠作为 疏水性苂光探针. 首先将 EC- $g$-PNIPAm 溶解于 $\mathrm{pH} 6.9$ 的超纯水中得到 $0.15 \mathrm{mg} / \mathrm{mL}$ 的溶液, 然后稀释得到 $1.5 \times 10^{-10} \sim 1.5 \times 10^{-1} \mathrm{mg} / \mathrm{mL}$ 的系列溶液, 待用. 溶液中 萠探针的浓度均为 $6.5 \times 10^{-5} \mathrm{~mol} / \mathrm{L}$. 激发波长为 $334 \mathrm{~nm}$, 记录萠第一个和第三个峰的苂光强度比与聚合物浓度 的关系，临界胶束浓度通过 Boltzmann 拟合后得到. 


\section{References}

[1] Gupta, K. C.; Sahoo, S. Biomacromolecules 2001, 2, 239.

[2] Braunecker, W. A.; Matyjaszewski, K. Prog. Polym. Sci. 2007, 32, 93.

[3] Hua, M.; Yang, W.; Xue, Q.; Chen, M. Q.; Liu, X. Y.; Yang, C. Acta Chim. Sinica 2005, 63, 631. (华慢, 杨伟, 薛乔, 陈明清, 刘晓亚, 杨成, 化学学报, 2005, 63, 631.)

[4] Dang, L.; Liu, S. X.; Yang, X.; Qi, X. J.; Wang, H. M.; Lu, H. Y.; Tian, R. Acta Chim. Sinica 2011，69，2755. (党莉, 刘守信, 杨犧, 齐晓君, 王红梅, 吕海燕, 田荣, 化学学报, 2011, 69, 2755.)

[5] Lei, Z. L.; Liu, Y. L. Acta Chim. Sinica 2006, 64, 2403. (雷忠利, 刘 亚兰, 化学学报, 2006, 64, 2403.)

[6] Qi, X. J.; Liu, S. X.; Liu, T.; Dang, L.; Yang, X.; Yu, W. N.; Wang, H. M. Acta Chim. Sinica 2011, 69, 1803. (齐晓君, 刘守信, 刘腾, 党莉, 杨曦, 雨薇娜, 王红梅, 化学学报, 2011, 69, 1803.)

[7] Zhu, Y. M.; Zhang, Y.; Liu, Z. L.; Lang, M. D. Acta Chim. Sinica 2010, 68, 2449. (朱亚明, 张琰, 刘子路, 郎美东, 化学学报, 2010, 68,2449 .)

[8] Zhao, F. B.; Liu, Z. L.; Feng, L.; Sun, J. P.; Hu, J. W. Acta Polym. Sinica 2009, 166. (赵发宝, 刘志雷, 冯良, 孙建平, 胡继文, 高分 子学报, 2009, 166.)

[9] Huang, X. W.; Gu, L. N.; Lu, G. L.; Huang, X.; Zhang, Y. Q.; Huang, X. Y. Acta Chim. Sinica 2009, 67, 1363. (黄晓炜, 顾丽娜, 陆国林, 黄啸, 张亚琴, 黄晓宇, 化学学报, 2009, 67, 1363.)

[10] Qu, G.; Jiang, F. S.; Zhang, S. Y. Acta Polym. Sin. 2007, 541 (in Chinese). (屈刚, 姜复松, 张姝妍, 高分子学报, 2007, (6), 541.)

[11] Lu, R. F.; Hu, J. W.; Zhang, G. W.; Xiao, D. S. Acta Polym. Sin. 2012, 239. (卢汝烽, 胡继文, 张干伟, 肖定书, 高分子学报, 2012, 239.)

[12] Yi, Z.; Xi, F.; Chen, Y. M. Acta Polym. Sin. 2011, 494. (伊卓, 习复, 陈永明，高分子学报, 2011, (5), 494.)

[13] Zhang, X. H.; Wang, B. D.; Yang, D.; Yuan, L.; Tang, Q. Q.; Hu, J. H. Chin. J. Chem. 2009, 27, 2273.

[14] Yang, T. T.; Xu, Y. Y.; Yao, L.; Peng, H.; Cheng, S. Y. Acta Polym. Sin. 2009, 1146. (杨婷婷, 徐莹莹, 姚丽, 彭慧, 程时远, 高分子 学报, 2009, 1146.)

[15] Hu, N.; Ni, Z. B.; Chu, H.; Liu, X. Y.; Chen, M. Q. Chin. J. Chem. 2009, 27, 2249.

[16] Peng, D.; Li, Y. G.; Zhang, X. H.; Lu, G. L.; Feng, C.; Huang, X. Y. Acta Chim. Sinica 2007, 65, 2144. (彭丹, 李圭功, 张晓环, 陆国 林, 冯纯, 黄晓宇, 化学学报, 2007, 65, 2144.)

[17] Huang, C. G.; Wan, X. L.; Ying, S. K. Acta Polym. Sin. 2000, 467. (黄昌国, 万小龙, 应圣康, 高分子学报, 2000, 467.)

[18] Sun, J. Y.; Huang, W. Y.; Xue, X. Q.; Jiang, B. B.; Zhai, G. Q.; Kong, L. Z.; Chen, J. H.; Yang, Y.; Zhang, D. L. Acta Polym. Sin. 2012, 749. (孙佳悦, 黄文艳, 薛小强, 蒋必彪, 翟光群, 孔立智, 陈建 海, 杨扬, 张东亮，高分子学报, 2012, 749.)

[19] Ge, Z.; Cai, Y.; Yin, J.; Zhu, Z.; Rao, J.; Liu, S. Langmuir 2006, 23, 1114.

[20] Yu, T.; Wang, Y.; Lu, D. R.; Bai, R. K.; Lu, W. Q. Acta Polym. Sin. 2006, 597. (于涛, 王等, 鲁代仁, 白如科, 陆伟琪, 高分子学报, 2006, 597.)

[21] Gao, H.; Matyjaszewski, K. Macromolecules 2006, 39, 3154.

[22] Westlund, R.; Carlmark, A.; Hult, A.; Malmstrom, E.; Saez, I. M. Soft Matter 2007, 3, 866 .

[23] Carlmark, A.; Malmstrom, E. J. Am. Chem. Soc. 2002, 124, 900.

[24] Carlmark, A.; Malmstrom, E. E. Biomacromolecules 2003, 4, 1740.

[25] Li, Q.; Kang, H.; Liu, R.; Huang, Y. Chin. J. Chem. 2012, 30, 2169.

[26] Vlcek, P.; Janata, M.; Latalova, P.; Kriz, J.; Cadova, E.; Toman, L. Polymer 2006, 47, 2587.

[27] Yan, Q.; Yuan, J. Y.; Kang, Y.; Yin, Y. W. Prog. Chem. 2010, 22, 449.

[28] Yuan, W. Z.; Zhang, J. C.; Zou, H.; Shen, T. X.; Ren, J. Polymer 2012, 53, 956

[29] Shen, D. W.; Yong, H. Polymer 2004, 45, 7091.

[30] Shen, D. W.; Yu, H.; Huang, Y. J. Polym. Sci. Part A Polym. Chem. 2005, 43, 4099.

[31] Yan, Q.; Yuan, J. Y.; Zhang, F. B.; Sui, X. F.; Xie, X. M.; Yin, Y. W.; Wang, S. F.; Wei, Y. Biomacromolecules 2009, 10, 2033.

[32] Meng, T.; Gao, X.; Zhang, J.; Yuan, J. Y.; Zhang, Y. Z.; He, J. S.
Polymer 2009, 50, 447.

[33] Yan, Q.; Yuan, J.; Kang, Y.; Yin, Y. Prog. Chem. 2010, 22, 449.

[34] Ostmark, E.; Harrisson, S.; Wooley, K. L.; Malmstrom, E. E. Biomacromolecules 2007, 8,1138 .

[35] Ostmark, E.; Lindqvist, J.; Nystrom, D.; Malmstrom, E. Biomacromolecules 2007, 8, 3815.

[36] Lindqvist, J.; Nystrom, D.; Ostmark, E.; Antoni, P.; Carlmark, A.; Johansson, M.; Hult, A.; Malmstrom, E. Biomacromolecules 2008, 9, 2139.

[37] Kang, H. L.; Liu, W. Y.; Liu, R. G.; Huang, Y. Macromol. Chem. Phys. 2008, 209, 424.

[38] Ma, L.; Kang, H. L.; Liu, R. G.; Huang, Y. Langmuir 2010, 26, 18519.

[39] Li, Y. X.; Liu, R. G.; Liu, W. Y.; Kang, H. L.; Wu, M.; Huang, Y. J. Polym. Sci. Part A Polym. Chem. 2008, 46, 6907.

[40] Ma, L.; Liu, R. G.; Tan, J. J.; Wang, D. Q.; Jin, X.; Kang, H. L.; Wu, M.; Huang, Y. Langmuir 2010, 26, 8697.

[41] Wang, D. Q.; Tan, J. J.; Kang, H. L.; Ma, L.; Jin, X.; Liu, R. G.; Huang, Y. Carbohydr. Polym. 2011, 84, 195.

[42] Liu, W. Y.; Liu, R. G.; Li, Y. X.; Kang, H. L.; Shen, D.; Wu, M.; Huang, Y. Polymer 2009, 50, 211.

[43] Liu, W. Y.; Liu, R. G.; Li, Y. X.; Wang, W.; Ma, L.; Wu, M.; Huang, Y. Polymer 2009, 50, 2716.

[44] Tan, J. J.; Li, Y. X.; Liu, R. G.; Kang, H. L.; Wang, D. Q.; Ma, L.; Liu, W. Y.; Wu, M.; Huang, Y. Carbohydr. Polym. 2010, 81, 213

[45] Zhang, Y. W.; Jiang, M. Acta Polym. Sin. 2005, 650.

[46] Dou, H. J.; Jiang, M.; Peng, H. S.; Chen, D. Y.; Hong, Y. Angew. Chem., Int. Ed. 2003, 42, 1516.

[47] Dou, H.; Jiang, M. Polym. Int. 2007, 56, 1206.

[48] Wan, S.; Jiang, M.; Zhang, G. Z. Macromolecules 2007, 40, 5552.

[49] Liu, W. Y.; Liu, Y. J.; Hao, X. H.; Zeng, G. S.; Wang, W.; Liu, R. G.; Huang, Y. Carbohydr. Polym. 2012, 88, 290.

[50] Oupický, D.; Reschel, T.; Koňák, Č.; Oupická, L. Macromolecules 2003, 36, 6863 .

[51] Ohya, S.; Nakayama, Y.; Matsuda, T. Biomacromolecules 2001, 2, 856.

[52] Chu, L. Y.; Zhu, J. H.; Chen, W. M.; Niitsuma, T.; Yamaguchi, T.; Nakao, S. Chin. J. Chem. Eng. 2003, 11, 269.

[53] Gu, J.; Xia, F.; Wu, Y.; Qu, X.; Yang, Z.; Jiang, L. J. Controlled Release 2007, 117, 396.

[54] Xie, G. M.; Song, X. Q.; Ma, Z. B.; Yang, C. Chem. Res. Appl. 2007, 19, 629. (解光明, 宋晓青, 马宗斌, 杨成, 化学研究与应用, 2007, 19, 629.)

[55] Li, X. J.; Yin, M. H.; Zhang, G. L.; Zhang, F. B. Chinese J. Chem. Eng. 2009, 17, 145 .

[56] Bokias, G.; Mylonas, Y.; Staikos, G.; Bumbu, G. G.; Vasile, C. Macromolecules 2001, 34, 4958.

[57] Li, H.; Luo, J.; Zhu, D.; Zhou, D.; Liang, L.; Lv, M. Fine Chem. 2010, 27, 968. (李欢, 罗建新, 朱东雨, 周德文, 梁利岩, 吕满庚, 精细化工, 2010, 27, 968.)

[58] Xia, Y.; Yin, X.; Burke, N. A. D.; Stöver, H. D. H. Macromolecules 2005, 38, 5937.

[59] Percec, V.; Guliashvili, T.; Ladislaw, J. S.; Wistrand, A.; Stjerndahl, A.; Sienkowska, M. J.; Monteiro, M. J.; Sahoo, S. J. Am. Chem. Soc. 2006, $128,14156$.

[60] Zhai, S.; Wang, B.; Feng, C.; Li, Y.; Yang, D.; Hu, J.; Lu, G.; Huang, X. J. Polym. Sci. Part A Polym. Chem. 2010, 48, 647.

[61] Feng, C.; Shen, Z.; Li, Y.; Gu, L.; Zhang, Y.; Lu, G.; Huang, X. J. Polym. Sci. Part A Polym. Chem. 2009, 47, 1811.

[62] Wu, C. Polymer 1998, 39, 4609.

[63] Li, Y.; Zhang, Y.; Yang, D.; Li, Y.; Hu, J.; Feng, C.; Zhai, S.; Lu, G.; Huang, X. Macromolecules 2010, 43, 262.

[64] Zhang, Y.; Shen, Z.; Yang, D.; Feng, C.; Hu, J.; Lu, G.; Huang, X. Macromolecules 2010, 43, 117.

[65] Li, M.; Jahed, N. M.; Min, K.; Matyjaszewski, K. Macromolecules 2004, 37, 2434

[66] Wu, T.; Zhang, Y.; Wang, X.; Liu, S. Chem. Mater. 2007, 20, 101.

[67] Schmidt, S.; Motschmann, H.; Hellweg, T.; von Klitzing, R. Polymer 2008, 49, 749.

[68] Strandman, S.; Luostarinen, M.; Niemela, K.; Tenhu, H.; Rissanen, K. J. Polym. Sci. Part A Polym. Chem. 2004, 42, 4189. 